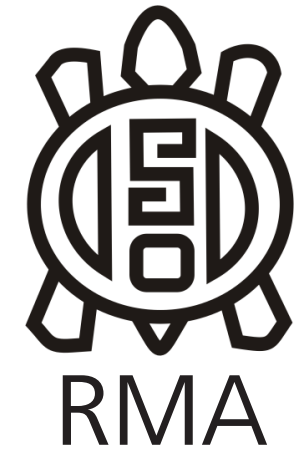

Dossier

\title{
Estudio de caso: conservación y análisis osteobiográfico del material óseo proveniente del sitio Hornos de Cal, costa de Taltal, norte de
} Chile

\author{
Case study: conservation and osteobiographic analysis of bone \\ material from the site Hornos de Cal, Costa de Taltal, north of Chile
}

Monserrat Araneda*y Pedro Andrade**

*Universidad de Concepción. E-mail: monaraneda96@gmail.com

**Carrera de Antropología, Universidad de Concepción. Programa de doctorado UTA-UCN. E-mail: pandradem@udec.c

\begin{abstract}
Resumen
El sitio Hornos de Cal corresponde a un conchero ubicado en la costa desértica de Antofagasta, a $16 \mathrm{~km}$ al sur de Taltal. Se trata de un contexto funerario conformado por el entierro de un individuo adulto sin ofrendas asociadas, datado en el $6180 \pm 25 \mathrm{cal}$ AP. El presente trabajo posee un enfoque osteobiográfico, donde a partir de estimaciones y determinaciones de sexo, la edad, y la estatura del individuo, como también patologías óseas, intentamos relacionar estos marcadores con su contexto temporal y cultural. Dada la presencia de rasgos particulares de este individuo que lo diferencian de su contexto poblacional, reflexionamos sobre la necesidad de considerar estos casos dentro de las reconstrucciones bioarqueológicas, ya que ayudan a comprender situaciones que dentro de un enfoque poblacional pueden pasar desapercibidas.
\end{abstract}

Palabras clave: Norte de Chile; Cazadores-Recolectores costeros; Osteobiografía; Patologías congénitas.

\begin{abstract}
:
The site Hornos de Cal corresponds to a shell midden located on the desert coast of Antofagasta, $16 \mathrm{~km}$ south of Taltal. It is a burial context made up of the burial of an adult individual with no associated offerings, dated to 6180$25 \mathrm{cal}$ AP. The present work has an osteobiographical approach, where we try to relate data on sex, age, height, and bone pathologies of the individual to their temporal and cultural context. Given the presence of particular traits of this individual that differentiate him from his population context, we reflect on the need to consider these cases within bioarcheological reconstructions, as they help to understand situations that within a population approach may go unnoticed.
\end{abstract}

Keywords: North of Chile; Coastal hunter-gatherers; Osteobiography; Sacralization of L5.

\section{Introducción}

Los estudios bioarqueológicos de la costa arreica son escasos, en comparación a las zonas costeras aledañas de los Valles Occidentales y del Norte Semiárido (Andrade, Salazar, Urrea y Castro, 2014). No obstante, los trabajos sistemáticos de diferentes equipos de investigación en la zona, durante los últimos 15 años (Cocilovo, Varela, Costa-Junqueira y Quevedo, 2005; Arias y Herrera, 2012; Constanzo, 2013; Berrios, 2014; Andrade et al., 2014; Andrade, Castro y Aldunate, 2016; entre otros), han permitido caracterizar y aproximarnos a los modos de vida de los habitantes pretéritos de este hostil sector. Así, dentro de una extensa cronología que se remonta hasta más allá de 12000 años de forma casi ininterrumpida (Salazar et al., 2018; San Francisco y Ballester, 2018), se puede dar cuenta de la presencia de poblaciones humanas que lograron permanecer con una subsistencia basada en la caza y recolección, principalmente de recursos marinos (Santana, Herrera y Uribe, 2012; Clarot y Ballester, 20142015; Andrade et al., 2015; Andrade et al., 2016; Pestle, Torres-Rouff, Gallardo, Ballester y Clarot, 2015a; Pestle et al., 2015b; Pestle, 2017).

El objetivo de la presente investigación es analizar ostebiográficamente los restos óseos humanos provenientes del sitio Hornos de $\mathrm{Cal}$, un conchero que presenta ocupaciones que abarcan entre los ca. 6500 y 3350 cal AP y que se encuentra al sur de la localidad de Taltal, en la región de Antofagasta. Para ello, se estimará el perfil biológico del individuo (sexo, edad, estatura, actividad ocupacional, patologías, traumatismos), y se correlacionarán los 
resultados con la evidencia arqueológica y bioantropológica que se posee sobre los habitantes de la costa de Taltal en este período. A partir de esto, queremos conocer aquellos rasgos propios del individuo que lo ligan con su población de origen, pero también reflexionar sobre la importancia de relevar características particulares del individuo analizado.

Si bien el concepto de osteobiografía ha tenido diversas interpretaciones en los últimos años, dependiendo además de los contextos disciplinares en donde se le utiliza (para una revisión histórica del concepto, véase Mihanovic et al., 2017), parece existir consenso en que su principal objetivo corresponde a la interpretación de la evidencia esquelética de un individuo determinado con respecto a su población de origen (Hamilakis, Pluciennik y Tarlow, 2002; Robb, 2002), perspectiva que compartimos. Ahora bien, dentro de este marco interpretativo, consideramos que las evidencias esqueléticas fundamentales para llevar a cabo este enfoque deben incluir determinaciones y/o estimaciones de sexo, edad, estatura, patologías, actividades habituales y adscripción cronológica y espacial del individuo en relación con su población de origen, tal como plantean Mihanovic y colaboradores (2017).

\section{Antecedentes ambientales y arqueológicos de la costa desértica de Antofagasta}

La costa desértica de Antofagasta corresponde a una franja territorial de casi $500 \mathrm{~km}$ de largo, que se extiende desde el sur de la desembocadura del río Loa, hasta el sur de la ciudad de Taltal. Esta franja, de promedio cercano a los 2 km de ancho (Niemeyer, González y Martínez-de los Ríos, 1996), se encuentra delimitada por el occidente por la presencia del océano Pacífico y por el oriente por el farellón costero montañoso, conocido localmente como Cordillera de la Costa, la cual puede alcanzar alturas de 1300 msnm (Ortega y Vargas, 2016) y un ancho que puede alcanzar los 80 km (Asún, 2017).

La franja costera que se produce entre estas dos barreras naturales conforma un ambiente extremadamente hostil, debido principalmente a la ausencia de cursos de agua, siendo las únicas fuentes de recurso hídrico, pequeñas y acotadas áreas de afloramiento de agua, conocida como aguadas (Herrera y Custodio, 2014). Esto, sumado a la presencia de una niebla costera, conocida en la zona como Camanchaca, que se transforma en una débil precipitación al entrar en contacto con la vertiente occidental de la Cordillera de la Costa, permiten sostener una frágil flora y la subsistencia de fauna (Guerra, 2004; Larraín, 2007).

Sumado a estas condiciones, la presencia de desastres naturales es recurrente en la zona, con un paleoregistro del fenómeno de El Niño - el cual causa torrenciales Iluvias y aluviones (Vargas, Ortlieb y Rutllant, 2000) como también la presencia de terremotos y tsunamis. Para el caso del primero, se ha identificado su presencia desde el Pleistoceno Tardío (Vargas et al., 2000; Meza, 2019), mientras que para los segundos, se ha registrado su ocurrencia desde al menos el Holoceno Medio (León et al., 2019).

A pesar de estas situaciones, el poblamiento humano en la costa de Antofagasta posee una larga data, pudiendo registrarse de manera casi continua desde hace unos 12000 años atrás (Salazar et al., 2015). Esto se debe, principalmente, a la presencia de un ambiente marino diverso y productivo (Ortlieb, Vargas y Saliege, 2011), el cual fue la base del sustento de las sociedades cazadoras-recolectoras-pescadoras que ocuparon este ambiente. La característica poblacional mencionada anteriormente, ha encontrado respaldo en el registro artefactual, zooarqueológico, bioarqueológico y espacial (Castelleti, 2007; Castro, Aldunate y Varela, 2012; Castro, 2014; Salazar et al., 2015; Andrade et al., 2014; Salazar et al., 2018; Salazar et al., 2020). Estudios realizados recientemente, sobre la apropiación cultural del territorio (para más detalles de este proceso, véase Salazar et al., 2018), indican que la adopción de este tradicional modo de vida cazador-recolector-pescador posee una larga data en la zona, manteniéndose de forma estable hasta momentos de la imposición colonial (Ballester, San Francisco y Gallardo, 2010; Andrade et al., 2014; Andrade et al., 2015).

En este escenario, la presencia de sociedades con una data tan larga, muestra grupos humanos que comienzan la especialización tecnológica enfocada en la extracción de recursos marinos hacia el $7500 \mathrm{cal}$ AP generando, con el tiempo, extensos concheros, complejos equipamientos para extracción de recursos marinos (Salazar et al., 2015; Salazar et al., 2020), junto con la expresión temprana de navegación (Olguín, Salazar y Jackson, 2014), lo cual les permitió acceder y conquistar las distintas dimensiones del ambiente marino (sensu Llagostera, 1989). Este momento, es conocido en la cronología local como Arcaico III y se extenderá hasta el 5500 cal AP (Salazar et al., 2015). Uno de los sitios que presenta una ocupación inicial asociada a este periodo corresponde a Hornos de Cal.

\section{El sitio Hornos de Cal y su contexto funerario}

El sitio Hornos de Cal (Salazar et al., 2020; Andrade y Araneda, 2020) se encuentra ubicado $16 \mathrm{~km}$ al sur de Taltal (Ver Figura 1) y corresponde a un conchero de unos $2000 \mathrm{~m}^{2}$. Los trabajos en terreno consistieron en la limpieza de perfiles de sectores que se encontraban saqueados, como también la excavación de una trinchera de $2 \times 1$ metros, siguiendo los estratos naturales, discriminados de manera artificial cada $5 \mathrm{~cm}$, en el marco del proyecto FONDECYT 1151203.

Esta excavación tuvo como resultado la identificación cultural y estratigráfica de tres ocupaciones, correspondientes a los períodos Arcaico III, IV y VI, siendo las dos primeras muy 


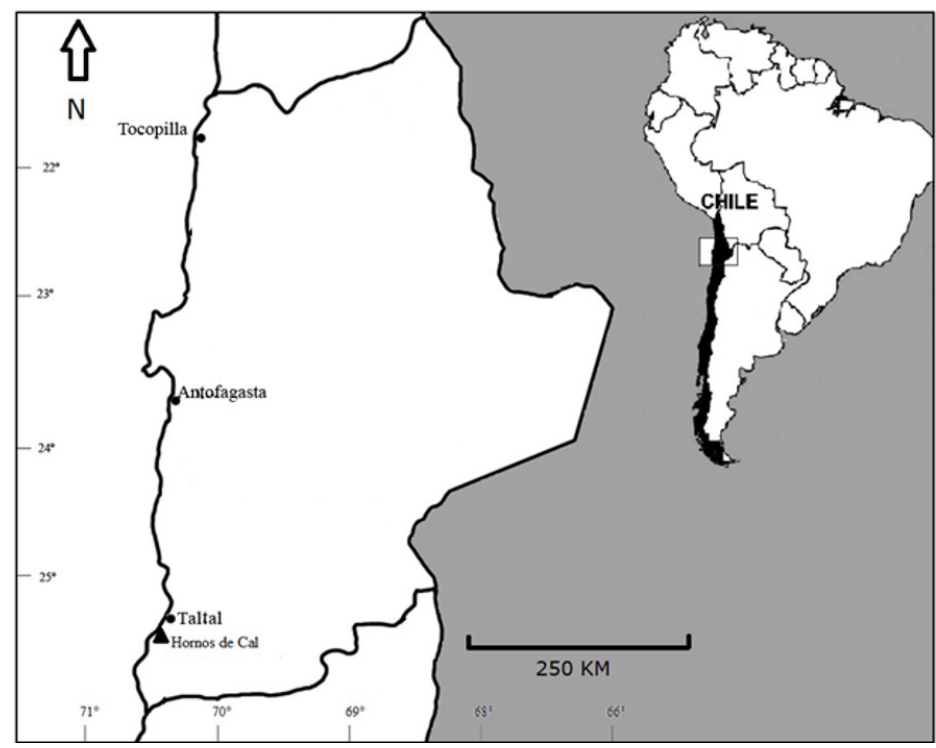

Figura 1: Ubicación geográfica del sitio arqueológico Hornos de Cal y de las cuidades de la costa de la región de Antofagasta.

Figure 1: Geographical location of archaeological site Hornos de Cal and cities in the coast of the Antofagasta region.

potentes, mientras que la tercera presenta características de una ocupación efímera (para conocer detalles de cada una de las ocupaciones, véase Salazar et al., 2020). Estas ocupaciones humanas se vieron confirmadas a partir de las fechas radiocarbónicas presentadas en la Tabla 1.

La primera ocupación del sitio, es decir, aquella correspondiente al Arcaico III (7500 al 5500 cal AP., sensu Salazar et al., 2015), se realizó directamente sobre un paleoplaya de conchillas, donde se registraron materiales culturales correspondientes a restos de talla lítica (para más detalles de esta ocupación, véase Salazar et al., 2020). Dentro de esta paleoplaya, se registró un contexto funerario, el cual corresponde a un individuo adulto que se encontraba en posición decúbito dorsal, con los miembros superiores flectados y los miembros inferiores hiperflectados e inclinados hacia la derecha. Este contexto no presentó ajuar ni ofrenda asociada (ver Figura 2), y presenta algunas similitudes con aquellos registrados por Costa-Junqueira (2001) en el sitio La Chimba 13.

\section{Materiales y métodos}

La evaluación del estado de conservación de los elementos óseos se llevó a cabo siguiendo los estándares y criterios de observación macroscópica, establecidos por Cordero (2018). Se realizaron labores de limpieza superficial, mediante el uso de brocha y pinceles en seco; extracción del material malacológico adherido a los huesos, removidos aplicando de manera localizada y específica una solución de agua destilada más alcohol al $70 \%$ con instrumental metálico fino; y reconstrucción de las piezas mediante acetato de polivinilo.

El sexo se determinó a partir de los parámetros morfológicos en coxal según Buikstra y Ubelaker (1994). No se utilizaron determinaciones de sexo en cráneo, ya que este elemento óseo se vio afectado al momento del descubrimiento del individuo, resultando altamente fragmentado. De la misma forma, debido al estado de conservación de los huesos largos, no se aplicaron determinaciones métricas existentes para la población chilena actual (Garrido-Varas, Thompson y Campbell, 2014) o argentina colonial (Mansegosa, Giannotti, Chiavazza y Barrientos, 2018). A esto, se debe sumar el hecho de la distancia tanto geográfica como cronológica de nuestro individuo en estudio en relación con las poblaciones utilizadas para la generación de estas estimaciones, lo cual nos puede llevar a tener un diagnóstico errado.

La edad se estimó por medio de la fusión de epífisis en los huesos largos y vértebras (Bass, 1987; Buikstra y Ubelaker, 1994), el desarrollo osteológico de las unidades óseas (Scheuer y Black, 2000) y el grado de desgaste dental de Guerasimov (1995) y Lovejoy (1985). Por su parte, la estatura se estimó a través del método de Genovés modificado por Del Ángel y Cisneros (2002) utilizando el largo máximo de la ulna, radio y fíbula derecha. Si bien existen ecuaciones de determinación de estatura para población chilena (Ross y Manneschi, 2011), estas consideran las medidas de elementos óseos que por razones de conservación no pudieron ser utilizadas en el presente estudio.

\begin{tabular}{|c|c|c|c|c|c|}
\hline Código Muestra & Material & Fecha Convencional (AP) & Fecha Calibrada AP (2б) & Asignación Cronológica Local & Referencia \\
\hline Beta- 169540 & Concha & $3780 \pm 80$ & $3348(3790-3136)$ & Arcaico VI & Castelleti, 2007 \\
\hline D-AMS024529 & Carbón & $3883 \pm 29$ & $4252(4408-4150)$ & Arcaico IV & Salazar et al., 2020 \\
\hline UGAMS 31193 & Carbón & $3990 \pm 20$ & $4412(4447-4291)$ & Arcaico IV & Salazar et al., 2020 \\
\hline UGAMS 31194 & Concha & $5750 \pm 25$ & $5910(6166-5675)$ & Arcaico III & Salazar et al., 2020 \\
\hline D-AMS024530 & Carbón & $5255 \pm 31$ & 5965 (6023-5905) & Arcaico III & Salazar et al., 2020 \\
\hline D-AMS024526 & Carbón & $5272 \pm 34$ & 5979 (6030-5911) & Arcaico III & Salazar et al., 2020 \\
\hline UGAMS 31192 & Carbón & $5560 \pm 25$ & $6312(6399-6278)$ & Arcaico III & Salazar et al., 2020 \\
\hline D-AMS024528 & Carbón & $5688 \pm 31$ & $6426(6497-6317)$ & Arcaico III & Salazar et al., 2020 \\
\hline D-AMS014527 & Carbón & $5813 \pm 30$ & $6572(6664-6479)$ & Arcaico III & Salazar et al., 2020 \\
\hline
\end{tabular}

Tabla 1: Fechas sitio Hornos de Cal (Castelleti, 2007; Salazar et al., 2020).

Table 1: Hornos de Cal site dates (Castelleti, 2007; Salazar et al., 2020). 


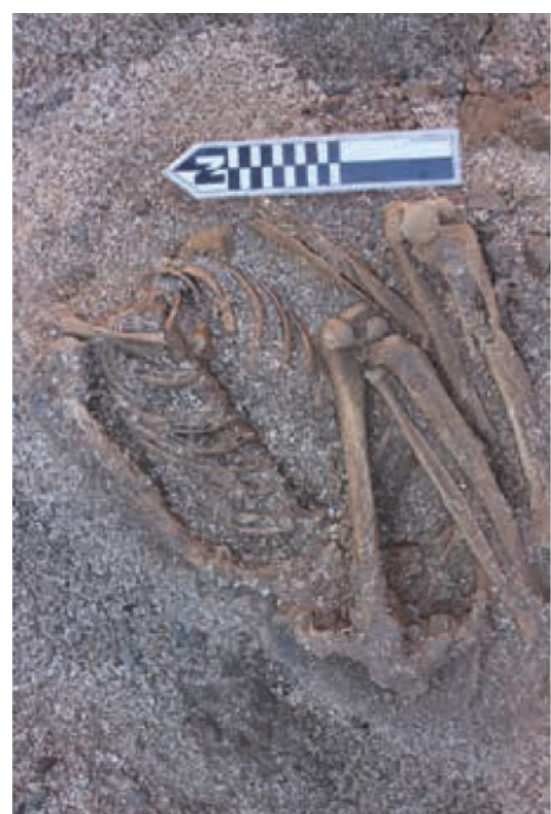

Figura 2: Contexto primario del entierro.

Figure 2: Primary context of burial.

Para el reconocimiento de las distintas condiciones patológicas y el grado de intensidad presente en los restos óseos se consideraron posibles eventos traumáticos, enfermedades degenerativas, infecciosas y nutricionales, siguiendo las indicaciones de Aufderheide y RodríguezMartín (1998), Buikstra y Ubelaker (1994) y Mann y Hunt (2005). En cuanto al análisis de las piezas dentales, el registro y determinación de patologías y desgaste, se realizó siguiendo las indicaciones de Chimeros (2003), sumado a la observación de rasgos dentales no métricos, según los parámetros de Rodríguez (2003).

Además, se envió una muestra ósea para la determinación de fechado radiocarbónico y de isótopos estables, los cuales fueron procesados en el Centro de Estudios Isotópicos Aplicados de la Universidad de Georgia,
Estados Unidos. Debido a la multiplicidad de ocupaciones que presenta el sitio, se consideró fundamental la obtención de este fechado directo, con el fin de acotar el escenario cronológico del individuo recuperado. La fecha obtenida fue calibrada aplicando la curva MARINE13 y el efecto reservorio para el norte de Chile propuesto por Ortlieb et al. (2011), considerando el alto consumo de recursos marinos que poseen los individuos de la zona (Andrade et al., 2015).

Finalmente, con respecto al enfoque osteobiográfico, se debe destacar que el individuo aquí presentado corresponde a uno de los más antiguos de la costa de Antofagasta, no existiendo registro de una población cronológicamente similar que nos permita caracterizarlo de forma comparativa dentro de un escenario poblacional mayor. Ahora bien, como se ha mencionado anteriormente, las poblaciones prehispánicas de la costa de Antofagasta y específicamente de la zona de Taltal se han caracterizado como cazadores-recolectores, utilizaremos como referencia comparativa las características generales que se han determinado para las poblaciones de la zona en diferentes periodos (Costa-Junqueira, 2001; Cocilovo et al., 2005; Ardiles, Ballester y Clarot, 2011; Andrade et al., 2014; Ballester, Clarot y Llagostera, 2014; Andrade et al., 2016; Mengozzi et al., 2016; Clarot y Ballester, 2014-2015; Torres-Rouff, 2017; Ballester et al., 2018; Castro, Pacheco, Kuzmanic, Clarot y Díaz, 2020).

\section{Resultados}

Si bien el esqueleto se encontraba mayoritariamente completo (ver Tabla 2), muchos elementos óseos se encuentran fragmentados. Por esta razón, se puede decir que el individuo analizado presenta un estado de conservación regular.

La determinación de sexo a través de la observación morfológica de los coxales indicó que se trata de un

\begin{tabular}{|l|c|l|c|}
\hline Elemento & Estado de conservación & Elemento & Estado de conservación \\
\hline Cráneo & 3 & Radio derecho & 3 \\
\hline Mandíbula & 3 & Radio izquierdo & 4 \\
\hline Maxilar & 4 & Mano derecha & 4 \\
\hline Hioides & 3 & Mano izquierda & 4 \\
\hline Esternón & 3 & Coxal derecho & 2 \\
\hline Escápula derecha & 3 & Coxal izquierdo & 2 \\
\hline Escápula izquierda & 3 & Sacro & 1 \\
\hline Clavícula derecha & 3 & Cóccix & 3 \\
\hline Clavícula izquierda & 2 & Fémur derecho & 3 \\
\hline Vértebras cervicales & 2 & Fémur izquierdo & 3 \\
\hline Vértebras dorsales & 3 & Patella derecha & 4 \\
\hline Vertebras lumbares & 3 & Patella izquierda & 4 \\
\hline Costillas derechas & 2 & Tibia derecha & 3 \\
\hline Costillas izquierdas & 2 & Tibia izquierda & 3 \\
\hline Húmero derecho & 3 & Fíbula derecha & 4 \\
\hline Húmero izquierdo & 1 & Fíbula izquierda & 2 \\
\hline Ulna derecho & 4 & Pie derecho & 4 \\
\hline Ulna izquierda & 4 & Pie izquierdo & 4 \\
\hline
\end{tabular}

Tabla 2: Registro osteológico según el grado de completitud de la pieza ósea (0: ausente, $1:<25 \%, 2: 25 \%$ 50\%, 3: 50\%-75\%, 4: >75\%).

Table 2: Osteological record according to the degree of completeness of the bone piece ( 0 : absent, $1:<25 \%, 2: 25 \%-50 \%, 3$ : $50 \%-75 \%, 4:>75 \%)$. 
individuo de sexo masculino. En cuanto a la estimación de edad, la suma de las variables analizadas y mencionadas en la sección anterior, señalan que el individuo en estudio se encontraría en un rango de entre 20 y 25 años de edad. Finalmente, sobre la estatura, las medidas obtenidas sobre los huesos largos disponibles muestran que el individuo habría alcanzado una estatura promedio de 151,25 $\pm 0,5$ $\mathrm{cm}$.

En cuanto a patologías y marcadores ocupacionales, se puede decir que este individuo presentaba casi una nula presencia de huellas de este tipo. De esta forma, se puede decir que el individuo no mostraba marcadores ocupacionales y/o degenerativas como porosidad, eburnación, lipping, exostosis, inflamación, crecimiento, entesopatías o pérdida ósea; o marcadores asociados a patologías metabólicas como hiperostosis porótica y/o criba orbitaria, o infecciosas. Similarmente, no presentaba huellas de lesiones traumáticas. En cuanto a marcadores ocupacionales, la evidencia esquelética muestra únicamente la presencia de facetas de acuclillamiento marcadas en ambas tibias y la presencia de vastus notch en ambas patellas.

En cuanto al análisis dental, se pudo disponer de la totalidad de las piezas. Sobre las piezas dentales superiores se puede decir que, debido al mal estado de conservación del cráneo, solo cinco dientes (ambos incisivos laterales, ambos segundo premolares y canino derecho) se encontraban in situ. Por su parte, de los dientes inferiores solo se pudieron registrar tres piezas en esta condición (ambos caninos y primer premolar derecho). En lo respectivo a la presencia de patologías, se destaca la ausencia total de caries y de hipoplasia del esmalte. Si fue posible observar la presencia de chipping en el $60 \%$ de las piezas analizadas, siendo principalmente afectadas las piezas anteriores del maxilar. Además, se pudo observar la depositación moderada de cálculo dental en todas las piezas. En cuanto al desgaste dental, en términos generales se observa moderado y plano, con

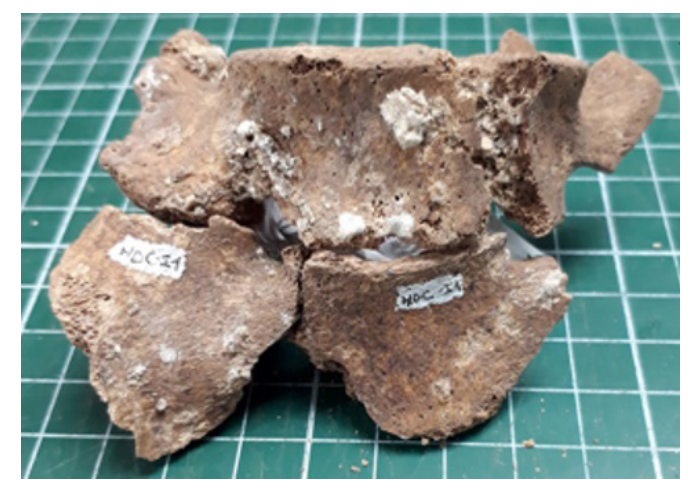

Figura 3: Vista anterior de fragmento de sacro con presencia de sacralización incompleta unilateral derecha de L5 (escala grilla: $1 \times 1 \mathrm{~cm}$ )

Figure 3: Previous view of sacral fragment with the presence of right unilateral incomplete sacralization of L5 (scale: $1 \times 1 \mathrm{~cm})$. una mayor afección en caninos, premolares y primeros molares. Finalmente, se puede decir que producto del análisis dental morfológico, se pudo determinar la presencia de diente en pala en incisivos y de la presencia de una quinta cúspide en el segundo molar inferior izquierdo. Ambos rasgos, se han asociado como determinantes de la asignación poblacional mongoloide (Hanihara, 1968; Turner, 1984).

A pesar de la ausencia de patologías morfofuncionales, degenerativas, metabólicas, nutricionales, etc., el individuo sí presenta una condición patológica llamativa en la zona lumbosacra. Esta corresponde a una hemisacralización unilateral derecha de L5 (ver Figura 3), una anomalía congénita poco frecuente que se produce debido a defectos en la segmentación de la columna lumbosacra durante el desarrollo (Murlimanju, Prabhu, Pai, Ganeshkumar y Sarvepalli, 2011).

Finalmente, se debe indicar que los análisis arqueométricos realizados sobre el individuo indican una fecha

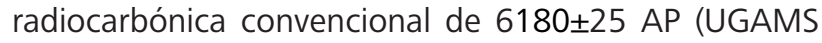
30567; $\delta^{13} C_{c}$ : -11.23; hueso humano), la cual luego de ser calibrada (6615 [6375] 6175 cal AP), lo sitúa dentro del periodo Arcaico III de la cronología local (Salazar et al., 2015). En lo respectivo a los análisis de isótopos estables del individuo, $13 \delta \mathrm{Ccol}:-11,23 \%$; $15 \delta \mathrm{Ncol}: 23,65 \%$, se puede apreciar que estos indican un alto consumo de proteínas marinas.

\section{Discusiones y conclusiones}

A partir de lo anteriormente expuesto, se puede decir que el individuo analizado al momento de ser comparado con las poblaciones prehispánicas de la zona de estudio presenta algunas similitudes, como también rasgos claramente distintivos.

Sobre las primeras, se puede decir que las condiciones paleopatológicas observadas en las piezas dentales, sumado a los resultados de los isótopos estables, nos muestra claramente que se trata de un individuo que mantuvo una dieta dura y/o rica en partículas abrasivas, como la arena, y basada en la ingesta de los abundantes recursos marinos locales, tal como se ha planteado en investigaciones previas en el área y ya mencionadas anteriormente. De manera similar la presencia de facetas de acuclillamiento en ambas tibias corresponden a un rasgo recurrente en los individuos recuperados en contextos de los diferentes periodos cronológicos de la costa desértica de Antofagasta, lo cual se ha ligado a actividades de extracción y procesamiento de recursos alimenticios y artefactuales (Andrade et al., 2014). Sin embargo, llama la atención la ausencia de patologías esqueletales que se aprecian de manera reiterada en las poblaciones prehispánicas de la zona de estudio, como aquellas degenerativas articulares y metabólicas (Andrade et al., 2014; Standen, Santoro y Arriaza, 2004; Andrade 
et al., 2016).

Ahora bien, dentro del espectro de las condiciones paleopatológicas presentes en el individuo destaca la hemisacralización unilateral derecha de L5. Tal como se expuso anteriormente, esta es una anomalía congénita que, según la clínica moderna, su prevalencia oscila entre el $4 \%$ y 35\%, dependiendo de la población en estudio (Otani, Konno y Kikuchi, 2001; Hsieh, Vanderford, Moreau y Prong, 1999; Leboeuf, Kimber y White, 1989; Vergauwen et al., 1997; Sekharappa, Amritanand, Krishnan y Samuel, 2014; entre otros). Esta baja manifestación se puede observar en la costa norte de Chile, reportándose además del individuo de Hornos de Cal, un caso en la zona de Valles Occidentales (Arriaza, Standen, Soto y Aravena, 2019) y otro en el sector de Caleta El Fierro, en la costa norte de la región de Antofagasta (Andrade y Araneda 2020).

Al respecto de la presencia de esta enfermedad podemos establecer dos interpretaciones que no son excluyentes entre sí. La primera de ella propone una relación con el origen de su expresión, marcada por una dimensión social y cultural de la patología. Tal como hemos planteado anteriormente (Andrade y Araneda, 2020), creemos que la manifestación de esta anomalía - por lo menos en la costa desértica de Antofagasta - se corresponde a uniones endogámicas llevadas a cabo por los grupos de este sector. Esta interpretación se encuentra sustentada por estudios etnohistóricos (Ballester y Gallardo, 2017; Ballester, 2017), osteológicos (Cocilovo et al., 2005; Cases, Rees, Pimentel, Labarca y Leiva, 2008; Ibacache, 2015; Andrade y Araneda, 2020) y genéticos (Henríquez, Moraga, Llop y Rothhammer, 2004; Rothhammer, Moraga, Santoro y Arriaza, 2010; de Saint-Pierre, Faure y Morales, 2020), que dan cuenta de la presencia de una práctica endogámica marcada en la zona de estudio, la cual se interrumpe, inicialmente en el siglo XVIII y posteriormente de manera sistemática desde la segunda mitad del siglo XIX en adelante (Letelier, 2016; Godoy, 2019; de Saint-Pierre et al., 2020).

Una segunda línea de interpretación corresponde al hecho de la ausencia de patologías morfofuncionales y degenerativas en el individuo recuperado del sitio Hornos de Cal. Tal como se ha mencionado anteriormente, este es un hecho atípico en las poblaciones de la costa de Antofagasta, las cuales de manera sostenida presentan este tipo de marcadores de actividad, relacionadas principalmente con la navegación y extracción de recursos marinos y mineros disponibles localmente (Castro et al., 2016; Salazar et al., 2015; Andrade et al., 2014; Andrade et al., 2016). Al respecto, se puede decir que estudios de individuos infantiles en la zona, indican que estos ingresarían a la vida productiva en una temprana edad (para más detalle, véase Loyola y Rebeco, 2011), lo cual se condice con las huellas observadas en individuos adultos.

Sobre este punto, es nuestra interpretación que el individuo de Hornos de Cal fue dejado al margen de actividades productivas intensas características de su grupo social más amplio, producto de su condición congénita, la cual no habría sido compatible con el desarrollo de estas. Si bien es cierto que existen reportes clínicos modernos de que la sacralización de L5 es asintomática (Elster, 1989; Barnes, 1994), también existen estudios de diferentes casos que indican justamente lo contrario, resaltando que esta condición puede causar dolor lumbar bajo y dolor glúteo (Tang et al., 2014; Nardo et al., 2012; Moreno, del Rio, Baltanás y Cía, 2016), cambios degenerativos en la columna vertebral (Hsieh et al., 1999; Leboeuf et al., 1989), una reducción en la altura del disco intervertebral (Hsieh et al., 1999) e incluso invalidez (Sahoo, Mohanty y Pattnaik, 2016). En el caso del individuo aquí analizado, la ausencia de los marcadores músculo-esqueletales recurrentes en las poblaciones de la costa desértica de Antofagasta, podría encontrar su explicación en este factor.

A la luz de esto y retomando la dimensión social de esta patología, estimamos que las poblaciones de la costa desértica de Antofagasta, podrían haber poseído una mirada diferenciada con respecto a aquellos individuos que se encontraban en una situación física disminuida con respecto a la generalidad del grupo, lo que no significó su aislamiento social dentro de este, tal como ha se ha reportado en otros casos para la costa del Norte Grande de Chile (Silva-Pinto, Valenzuela y Sepúlveda, 2013), sector donde además se ha observado que los traumas sufridos por los habitantes de la zona recibieron algún grado de tratamiento (Standen y Arriaza, 1999; Andrade et al., 2014; Andrade et al., 2016; Torres-Rouff, 2017).

Finalmente, si bien el enfoque osteobiográfico, tal como se entiende a partir de la literatura revisada, es útil para conocer la realidad del individuo analizado, presenta limitantes en cuanto la interpretación dentro de su contexto social mayor. Esto último se puede observar claramente, al dar cuenta de la presencia de una enfermedad prácticamente desconocida en la zona y la ausencia de aquellos marcadores osteológicos más característicos de los habitantes de la costa desértica de Antofagasta. Desde esta perspectiva, consideramos que, si bien la aproximación poblacional de los análisis bioarqueológicos presentan un alto potencial al momento de caracterizar de forma general a un grupo humano, se debe considerar igual de importante aquellos casos particulares, como el aquí reportado. Al realizar este tipo de ejercicios, creemos que podemos acceder a esferas interpretativas de manifestaciones sociales que muchas veces quedan ocultas dentro de la generalidad poblacional.

Concepción, 12 de mayo 2020

\section{Agradecimientos}

Se agradece al equipo perteneciente al proyecto Fondecyt 
1151203: "Trayectoria histórica, cambios ambientales y eventos catastróficos durante el Período Arcaico en la costa de Taltal, norte de Chile" cuyo investigador responsable es Diego Salazar, junto a César Borie, Carola Flores, Laura Olguín y Gabriel Vargas como coinvestigadores. Finalmente, ambos autores agradecen los valiosos comentarios de los evaluadores, los cuales ayudaron a mejorar la calidad de nuestro escrito.

\section{Bibliografía}

Andrade, P., Salazar, D., Urrea, J. y Castro, V. (2014). Modos de vida de los cazadores recolectores de la costa arreica del Norte Grande de Chile: una aproximación bioarqueológica a las poblaciones prehistóricas de Taltal. Chungará, 46(3), 467-491. http://dx.doi.org/10.4067/S0717-73562014000300010

Andrade, P., Fernández, R., Codjambassis, K., Urrea, J., Olguín, L., Rebolledo, S., Lira, F., Aravena, C. y Berrios, M. (2015). Subsistence continuity linked to consumption of marine protein in the formative period in the interfluvic coast of Northern Chile: re-assessing contacts with agropastoral groups from highlands. Radiocarbon, 57(4), 679-688. https://doi.org/10.2458/ azu_rc.57.18448

Andrade, P., Castro, V. y Aldunate, C. (2016). Reconstrucción del modo de vida de individuos del Arcaico de la costa arreica del Norte de Chile: una aproximación bioarqueológica desde el sitio Copaca 1. Chungará, 48(1), 73-90. http://dx.doi.org/10.4067/ S0717-73562016005000007

Andrade, P. y Araneda, M. (2020). Causas o azares: reflexiones en torno a la presencia de malformaciones congénitas de la columna vertebral en poblaciones arqueológicas de la costa desértica de Antofagasta, Norte de Chile. Revista Argentina de Antropología Biológica, 22(2). http://dx.doi. org/10.24215/18536387e019

Ardiles, H., Ballester, B. y Clarot, A. (2011). Elección de dieta en poblaciones pasadas de la costa de la II Región: una mirada multidisciplinaria. En Informes del Fondo de Apoyo a la Investigación Patrimonial (pp. 83-110). Dirección de Bibliotecas, Archivos y Museos de Santiago- Servicio Nacional del Patrimonio Cultural.

Arias, M. y Herrera, M. (2012). Caracterización del modo de vida, salud y dieta de las poblaciones de los cementerios CaseronesTarapacá 40 y Cáñamo 3 (Período Formativo, región de Tarapacá). En Actas del XVIII Congreso Nacional de Arqueología Chilena (pp. 45-54). Sociedad Chilena de Arqueología.

Arriaza, B., Standen, V., Soto, L. y Aravena, N. (2019). A prehistoric case of developmental defects of the spine in a young adult female from the Atacama Desert. International Journal of Osteoarchaeology, 29(2), 346-349. https://doi. org/10.1002/oa.2738

Asún, M. (2017). Análisis del registro histórico de catástrofes geológicas: evaluación del peligro remociones en masa para Taltal, Región de Antofagasta [tesis de pregrado, Universidad de Chile]. Repositorio académico de la Universidad de Chile. http://repositorio.uchile.cl/handle/2250/146692

Aufderheide, A. y Rodríguez Martin, C. (1998). The Cambridge Encyclopedia of Human Paleopathology. Cambridge University Press.

Ballester, B., San Francisco, A. y Gallardo, F. (2010). Modo de vida y economía doméstica de las comunidades cazadoras recolectoras costeras del Desierto de Atacama en tiempos coloniales y republicanos. Taltalia, 3, 21-32.

Ballester, B., Clarot, A. y Llagostera, A. (2014). El cementerio de Autoclub de Antofagasta y la sociedad litoral entre los 1000 y 1450 d.C. Hombre y Desierto, 18, 187-212.

Ballester, B. (2017). Parentesco y política de los cazadores, pescadores y recolectores marinos del Desierto de Atacama según crónicas, documentos históricos y restos materiales. En F. Gallardo, B. Ballester y N. Fuenzalida (Eds), Monumentos Funerarios en la Costa del Desierto de Atacama. Los cazadoresrecolectores marinos y sus intercambios (500 a.C. - 700 d.C.) (pp. 47-51). Serie Monográfica de la Sociedad Chilena de Arqueología $\mathrm{N}^{\circ} 7$.

Ballester, B. y Gallardo, F. (2017). La versatilidad del parentesco en la reproducción social: el caso de los cazadores-pescadores marinos del desierto de Atacama (siglos XVI-XIX, norte de Chile). Boletín de la Sociedad Chilena de Arqueología, 47, 7-28.

Ballester, B., Vidal, E., Calás, E., Gallardo, F., Aguilera, P., Pellegrino, C. y Clarot, A. (2018). Un enclave arcaico tardío en la aguada costera de Guala Guala (desierto de Atacama, Norte de Chile). Chungará, 50(3), 349-367. http://dx.doi.org/10.4067/ S0717-73562018005000702

Barnes, E. (1994). Developmental Defects of the Axial Skeleton in Paleopathology. University Press of Colorado.

Bass, W. (1987). Human Osteology: A Laboratory and Field Manual of the Human Skeleton. Missouri Archeological Society.

Berrios, M. (2014). Paleopatologías degenerativas de la Columna Vertebral de Restos Bioantropológicos de CazadoresRecolectores provenientes de la Costa Arreica. Comparación con una colección similar de la Costa del Norte Semiárido [tesis de pregrado, Universidad de Concepción]. Repositorio ANID. http://repositorio.conicyt.cl/handle/10533/189292

Buikstra, J. y Ubelaker, D. (1994). Standards for Data Collection from Human Skeletal Remains. Arkansas Archaeological Survey Research Series 44.

Cases, B., Rees, C., Pimentel, G., Labarca, R. y Leiva, D. (2008). Sugerencias desde un contexto funerario en un "espacio vacío" del Desierto de Atacama. Boletín del Museo Chile de Arte 
Precolombino, 13(1), 51-70. http://dx.doi.org/10.4067/S071868942008000100004

Castelleti, J. (2007). Patrón de asentamiento y uso de recursos a través de la secuencia ocupacional prehispana en la costa de Taltal [tesis de magister, Universidad Católica del NorteUniversidad de Tarapacá].

Castro, V., Aldunate, C. y Varela, V. (2012). Paisajes culturales de Cobija, costa de Antofagasta, Chile. Revista Chilena de Antropología, 26, 97-128. http://dx.doi.org/10.5354/07191472.2013 .26556

Castro, V. (2014). Pre-Hispanic cultures in the Atacama Desert: a Pacific coast overview. En N. Sanz, B. Arriaza y V. Standen (Eds), The Chinchorro Culture: A Comparative Perspective. The archaeology of the earliest human mummification (pp. 11-34). UNESCO - Universidad de Tarapacá.

Castro, V., Aldunate, C., Varela, V., Olguín, L., Andrade, P., García, F., Rubio, F., Castro, P., Maldonado, A. y Ruz, J. (2016). Ocupaciones arcaicas y probables evidencias de navegación temprana en la costa arreica de Antofagasta, Chile. Chungará, 48(4), 503-530. http://dx.doi.org/10.4067/S071773562016005000039

Castro, M., Pacheco, A., Kuzmanic, I., Clarot, A. y Díaz, P. (2020). Treponematosis in a pre-Columbian hunter-gatherer male from Antofagasta (1830 \pm 20 BP, Northern Coast of Chile). International Journal of Paleopathology, 30, 10-16. https://doi. org/10.1016/j.ijpp.2020.01.004

Chimeros, E. (2003). Perspectiva odontoestomatológica. En A. Isidro y A. Malgosa (Eds), Paleopatología. La enfermedad no escrita (pp.151 - 162). Editorial Mason.

Clarot, A. y Ballester, B. (2014-2015). Un costeño del desierto de Atacama: acercamiento al modo de vida en el periodo intermedio tardío mediante imagenología e isótopos estables. Taltalia, 7-8, 39-60.

Cocilovo, J., Varela, H., Costa-Junqueira, M. y Quevedo, S. (2005). Los pescadores arcaicos de la desembocadura del río Loa (norte de Chile): el sitio Caleta Huelén 42. Chungará, 37(1), 5-19. http://dx.doi.org/10.4067/S0717-73562005000100002

Constanzo, D. (2013). Una aproximación a la reconstrucción de los modos de vida a partir de las enfermedades degenerativas articulares en la columna vertebral en las poblaciones de la costa arreica en el norte grande de Chile [tesis de pregrado, Universidad de Concepción].

Cordero, L. (2018). Protocolo de manejo de colecciones y sistemas de inventario. Subdirección Nacional de Gestión Patrimonial-Centro de Documentación de Bienes Patrimoniales.

Costa-Junqueira, M. (2001). Modalidades de enterramientos humanos arcaicos en el norte de chile. Chungará, 33(1), 55-62.

\section{http://dx.doi.org/10.4067/S0717-7356200100010000}

De Saint Pierre, M., Faure, M. y Morales, H. (2020). Análisis histórico genético de la población de la región de Antofagasta revela discontinuidad en la costa y valles interiores. Estudios Atacameños, 64,183-198. http://dx.doi.org/10.22199/ issn.0718-1043-2020-0007

Del Angel, A. y Cisneros, H. (2004). Technical note: modification of regression equations used to estimate stature in Mesoamerican skeletal remains. American Journal of Physical Anthropology, 125(3), 264-265. https://doi.org/10.1002/ajpa.10385

Elster, A. (1989). Bertolotti's syndrome revisited. Transitional vertebrae of the lumbar spine. Spine, 14(12),1373-1377.

Garrido-Varas, C., Thompson, T. y Campbell, A. (2014). Parámetros métricos para la determinación de sexo en restos esqueletales chilenos modernos. Chungará, 46(2), 285-293. http://dx.doi.org/10.4067/S0717-73562014000200009

Godoy, M. (2019). Minería, crisis económicas y flujos migratorios en atacama meridional: Taltal (Chile), 1870-1950. Revista Historia, 26(2), 5-32. http://dx.doi.org/10.4067/S0717-88322019000200005

Guerasimov, M. (1995). The face finder. Lippincott.

Guerra, C. (2004). Fauna de vertebrados, flora y vegetación de la Desembocadura del río Loa. I y II Región de Chile. Centro Regional de Estudios y Educación Ambiental, Universidad de Antofagasta.

Hamilakis, Y., Pluciennik, M. y Tarlow, S. (Eds.). (2002). Thinking through the body: archaeologies of corporeality. Springer Science \& Business Media.

Hanihara, K. (1968). Mongoloid dental complex in the permanent dentition. En B. Endo, H. Hoshi y S, Masuda (Eds), Proceeding of the VIII International Symposium of Anthropological an Ethnological Science. Tokyo and Kyoto. Science Council of Japan.

Henríquez, B., Moraga, H., Llop, E. y Rothhammer, E. (2004). Caracterización genético molecular de habitantes de Caleta Paposo, último reducto Chango en Chile. Revista Médica de Chile, 132(6), 663-672. http://dx.doi.org/10.4067/S003498872004000600002

Herrera, C. y Custodio, E. (2014). Origins of waters from small springs located at the northern coast of Chile, in the vicinity of Antofagasta. Andean Geology, 41(2), 314-341. http://dx.doi. org/10.5027/andgeoV41n2-a03

Hsieh, C., Vanderford, J., Moreau, S. y Prong, T. (1999). Lumbosacral transitional segments: classification, prevalence, and effect on disk height. Journal of Manipulattive and Physiological Therapeutics, 23(7), 483-489. https://doi. org/10.1067/mmt.2000.108817 
Ibacache, M. (2015). Aproximación a estudio de los rasgos craneométricos de la costa arreica del norte de Chile [tesis de pregrado, Universidad de Concepción].

Larraín, B. (2007). Relaciones florísticas entre oasis de niebla del desierto costero del norte de Chile [tesis de pregrado, Universidad de Chile].

Leboeuf, C., Kimber, D. y White, K. (1989). Prevalence of spondylolisthesis, transitional anomalies and low intercrestal line in a chiropractic patient population. Journal of Manipulative and Physiological Therapeutics, 12(3), 200-204.

León, T., Vargas, G., Salazar, D., Goff, J., Guendon, L., Andrade, P. y Álvarez, G. (2019). Geo- archaeological records of large Holocene tsunamis along hyperarid coastal Atacama Desert in the major northern Chile seismic gap. Quaternary Science Review, 220, 335-358. https://doi.org/10.1016/j. quascirev.2019.07.038

Letelier, J. (2016). Entre la costa de Cobija y tierras altas. El tráfico arriero a inicios de la república boliviana. Diálogo Andino, 49, 225-234. http://dx.doi.org/10.4067/S071926812016000100022

Llagostera, A. (1989). Caza y pesca marítima. 9.000 a 1.000 a.C. Culturas de Chile Prehistoria. En C. Aldunate y I. Solimano (Eds), Desde sus Orígenes hasta los Albores de la Conquista (pp. 57-79). Editorial Andrés Bello.

Lovejoy C. (1985). Dental wear in the Libben Population: its functional pattern and role in the determination of adult skeletal age at death. American Journal of Physical Anthropology, 68(1), 47-56. https://doi.org/10.1002/ajpa.1330680105

Loyola, A. y Reveco, N. (2011). Bioarqueología de la Infancia y Marcadores Ocupacionales en la Costa Arreica Central en el Norte Grande de Chile [tesis de pregrado, Universidad de Concepción].

Mann, R. y Hunt, D. (2005). Photographic Atlas of bone disease. A guide to pathological and normal variation in the human skeleton. Charles C. Thomas Publishers.

Mansegosa, D., Giannotti, P., Chiavazza, H. y Barrientos, G. (2018). Funciones discriminantes para estimar sexo a partir de huesos largos en poblaciones coloniales del centro oeste de Argentina. Chungará, 50(1), 155-164. http://dx.doi. org/10.4067/S0717-73562017005000113

Mengozzi, F., Contreras, R., Andrade, P., Power, X., Flores, C., Salazard, D., Bravo, G., Urrea, J., Fernandes, R. y Hamman, C. (2016). Reconstrucción de un contexto funerario disturbado: artefactos y ecofactos del sitio El Gaucho (2325 cal AP), costa arreica del norte de Chile. Taltalia, 9, 7-25. https://doi. org/10.5281/zenodo.3780015

Meza, C. (2019). Aluviones históricos y prehistóricos en la ciudad de Taltal, Región de Antofagasta [tesis de pregrado, Universidad de Chile]. Repositorio académico de la Universidad de Chile. http://repositorio.uchile.cl/handle/2250/174186

Mihanovic, F., Jerkovic, I., Kruzic, I., Andelinovic, S., Jankovic, S. y Basic, Z. (2017). From biography to osteobiography: An example of anthropological historical identification of the remains of St. Paul. The Anatomical Record, 300(9), 1535-1546. https://doi. org/10.1002/ar.23602

Moreno, S., del Rio, P., Baltanás, P. y Cía, P. (2016). Síndrome de Bertolotti: a propósito de un caso. Revista Colombiana de Reumatología, 23(3), 200-203. https://doi.org/10.1016/j. rcreu.2015.12.007

Murlimanju, B., Prabhu, L., Pai, M., Ganeshkumar, C. y Sarvepalli, A. (2011). Lumbosacral transitional vertebrae: a case report and clinical implications. International Journal of Morphology, 29(4), 1123-1125. http://dx.doi.org/10.4067/ S0717-95022011000400008

Nardo, L., Alizai, H., Virayavanich, W., Lui, F., Hernandez, A., Lynch, J., Nevitt, C., Lane, N. y Link, T. (2012). Lumbosacral transitional vertebrae: association with low back pain. Radiology, 265(2), 497-503. https://doi.org/10.1148/radiol.12112747

Niemeyer, H., González, G. y Martínez-de los Ríos, E. (1996). Evolución tectónica cenozoica del margen continental activo de Antofagasta, Norte de Chile. Revista Geológica de Chile, 23(2), 165-186. http://dx.doi.org/10.5027/andgeoV23n2-a05

Olguín, L., Salazar, D. y Jackson, D. (2014). Tempranas evidencias de navegación y caza de especies oceánicas en la costa pacífica de Sudamérica (Taltal, 7.000 años cal. a.p.). Chungará, 46(2), 177-192. http://dx.doi.org/10.4067/S071773562014000200002

Ortega, C. y Vargas, G. (2016). Estimación de la tasa de erosión holocena en el escarpe costero de Antofagasta, Norte Grande de Chile. En Actas del XI Congreso Chileno Geológico (pp.117120). Universidad Católica del Norte.

Ortlieb, L., Vargas, G. y Saliege, J. (2011). Marine radiocarbon reservoir effect along the northern Chile-southern Peru coast $\left(14-24^{\circ} \mathrm{S}\right)$ throughout the Holocene. Quaternary Research, 75(1), 91-103. https://doi.org/10.1016/j.yqres.2010.07.018

Otani, K., Konno, S. y Kikuchi, S. (2001). Lumbosacral transitional vertebra and nerve root symptoms. The Journal of Bone and Joint Surgery, 83(8), 1137-1140. https://doi.org/10.1302/0301$620 \times .83 B 8.0831137$

Pestle, W., Torres-Rouff, C., Gallardo, F., Ballester, B. y Clarot, A. (2015a). Mobility and exchange among marine huntergatherer and agropastoralist communities in the Formative period Atacama Desert. Current Anthropology, 56(1), 121-133. https://doi.org/10.1086/679594 
Pestle, W., Torres-Rouff, C., Hubbe, M., Santana, F., Pimentel, G., Gallardo, F. y Knudson, K. (2015b). Explorando la diversidad dietética en la prehistoria del desierto de Atacama: un acercamiento a los patrones regionales. Chungará, 47(2), 201209. http://dx.doi.org/10.4067/S0717-73562015005000013

Pestle, W. (2017). Living, eating and dying in formative period Atacama Desert. En F. Gallardo, B. Ballester y N. Fuenzalida (Eds), Monumentos funerarios de la costa de desierto de Atacama. Los cazadores-recolectores marinos y sus intercambios $(500$ a.C. - 700 d.C.) (pp. 209-224). Series Monográficas de la Sociedad Chilena de Arqueología $N^{\circ} 7$.

Robb, J. (2002). Time and biography. En Y. Hamilakis, M. Pluciennik y S. Tarlow (Eds). Thinking through the Body (pp. 153-171). Springer Science \& Business Media.

Rodríguez, J. (2003). Dientes y diversidad humana, avances de la antropología dental. Editora Guadalupe.

Ross, A. y Manneschi, M. (2011). New identification criteria for the Chilean population: estimation of sex and stature. Forensic Science International, 204(1-3), 206.e1-206.e3. https://doi. org/10.1016/j.forsciint.2010.07.028

Rothhammer, F., Moraga, M., Santoro, C. y Arriaza, B. (2010). Origen de los Changos: análisis de ADNmt antiguo sugiere descendencia de pescadores de la cultura Chinchorro (7.9004.000 AP). Revista Médica de Chile, 138(2), 251-256. http:// dx.doi.org/10.4067/S0034-98872010000200016

Sahoo, P., Mohanty, P. y Pattnaik, M. (2016). Sacralization and herniated nucleus pulposus -An association study. Journal of Spine, 5(2), 2-7. http://dx.doi.org/10.4172/2165-7939.1000297

Salazar, D., Figueroa, V., Andrade, P., Salinas, H., Olguín, L., Power, X., Rebolledo, S., Orellana, H. y Urrea, J. (2015). Cronología y organización económicas de las poblaciones arcaicas de la costa de Taltal. Estudios Atacameños, 50, 7-46. http://dx.doi.org/10.4067/S0718-10432015000100002

Salazar, D., Arenas, C., Andrade, P., Olguín, L., Torres, J., Flores, C., Vargas, G., Rebolledo, S., Borie, C., Sandoval, C., Silva, C., Delgado, A., Lira, N. y Robles, C. (2018). From the use of space to territorialisation during the Early Holocene in Taltal, coastal Atacama Desert, Chile. Quaternary International, 473, 225-241. https://doi.org/10.1016/j.quaint.2017.09.035

Salazar, D., Power, X., Andrade, P., Flores, C., Rebolledo, S., Torres, J., Vargas, G., Monroy, I., Borie, C., Olguín, L. y Guendón, J. (2020). Trayectoria histórica y complejidad entre los cazadoresrecolectores-pescadores del Holoceno medio en la costa norte de Chile. En L. Sanhueza, A. Troncoso y R. Campbell (Eds), Iguales pero diferentes: Trayectorias históricas prehispánicas en el Cono Sur (pp. 49-94). Social-Ediciones.

San Francisco, A. y Ballester, B. (2018). Antiguos aleros al norte de Paposo. Taltalia, 11, 7-35.

Santana, F., Herrera, M. J. y Uribe, M. (2012). Acercamiento a la paleodieta en la costa y quebradas tarapaqueñas durante el período Formativo: análisis de isótopos estables a partir de tres casos de estudio. Boletín de la Sociedad Chilena de Arqueología, 41-42, 109-126.

Scheuer, L. y Black, S. (2000). Developmental juvenile osteology. Academic Press.

Sekharappa, V., Amritanand, R., Krishnan, V. y Samuel, K. (2014). Lumbosacral transition vertebra: prevalence and its significance. Asian Spine, 8(1), 51-58. https://doi.org/10.4184/asj.2014.8.1.51

Silva-Pinto, V., Valenzuela, D. y Sepúlveda, M. (2013). Paleopatología osteoarticular en Chinchorro: revisión de un caso y discusión sobre el autocuidado en la prehistoria de Arica, norte de Chile. Revista Médica de Chile, 141(5), 637-643. http:// dx.doi.org/10.4067/S0034-98872013000500012

Standen, V. y Arriaza, B. (1999). Traumas en las poblaciones Chinchorro (Costa Norte de Chile): ¿Violencia o situaciones accidentales? Chungará, 29(1), 133-150.

Standen, V., Santoro, C. y Arriaza, B. (2004). Síntesis y propuestas para el periodo arcaico en la costa del extremo Norte de Chile. Chungará, 36, 201-212. http://dx.doi.org/10.4067/ S0717-73562004000300023

Tang, M., Yang, X., Yang, S., Han, P., Ma, Y., Yu, H. y Zhu, B. (2014). Lumbosacral transitional vertebra in a population-based study of 5860 individuals: Prevalence and relationship to low back pain. European Journal of Radiology, 83(9), 1679-1682. https://doi.org/10.1016/j.ejrad.2014.05.036

Torres-Rouff, C. (2017). Fragmentos del cuerpo: una perspectiva bioarqueológica sobre la vida de personas en la Región de Antofagasta (cementerios de túmulos). En F. Gallardo, B. Ballester y N. Fuenzalida (Eds), Monumentos funerarios de la costa de desierto de Atacama. Los cazadores-recolectores marinos y sus intercambios (500 a.C. - 700 d.C.) (pp. 199-208). Series Monográficas de la Sociedad Chilena de Arqueología N 7.

Turner II, C. (1984). Advances in the Dental search for Native American Origins. Acta Anthropogenetica, 8(1-2), 23-78.

Vargas, G., Ortlieb, L. y Rutllant, J. (2000). Aluviones históricos en Antofagasta y su relación con eventos El Niño/Oscilación del Sur. Revista Geológica de Chile, 27(2), 157-176. http://dx.doi. org/10.4067/S0716-02082000000200002

Vergauwen, S., Parizel, P., Van Breusegem, L., Van Goethem, J., Nackaerts, Y., Van den Hauwe, L. y De Schepper, A. (1997). Distribution and incidence of degenerative spine changes in patients with a lumbo-sacral transitional vertebra. European Spine Journal 6(3), 168-172. https://doi.org/10.1007/BF01301431 